\title{
Sensor management of LEO constellation based on covariance control
}

\author{
QIN Zheng and LIANG Yan'gang* \\ College of Aerospace Science and Engineering, National University of Defense Technology, Changsha 410073, China
}

\begin{abstract}
This paper studies the multi-sensor management problem for low earth orbit (LEO) infrared warning constellation used to track a midcourse missile. A covariance control approach, which selects sensor combinations or subset based on the difference between the desired covariance matrix and the actual covariance of each target, is used for sensor management, including some matrix metrics to measure the differentia between two covariance matrices. Besides, to meet the requirements of the space based warning system, the original covariance control approach is improved. Simulation results demonstrate that the covariance control approach is able to provide a better tracking performance by providing a well-designed desired covariance and balance tracking performance goals with system demands.
\end{abstract}

Keywords: sensor management, covariance control, target tracking accuracy, LEO constellation.

DOI: $10.21629 /$ JSEE.2019.02.17

\section{Introduction}

In order to effectively deal with the threat of ballistic missiles, early warning systems have achieved rapid development in recent years $[1-10]$. Compared to the high altitude constellation, the low earth orbit (LEO) infrared warning constellation can effectively compensate for the observation gap to the missile target, thereby achieving the fullscale and continuous tracking of the target. However, due to the wide distribution of the LEO constellation, the highspeed movement of the target and limited sensor resources, it is often difficult for a single satellite to complete the tracking of the target. It is necessary to use multi-satellite to complete the tracking task. Thus, a sensor management method should be used to allocate limited sensor resources to the target to be tracked. Therefore, we use sensors located on the LEO satellite constellation to study the problem of multi-sensor management for tracking a midcourse

\footnotetext{
Manuscript received January 19, 2018.

*Corresponding author.

This work was supported by the National Natural Science Foundation of China (61690210; 61690213).
}

ballistic missile.

The core problem of sensor management is to establish an easy-to-quantize objective function according to certain criteria, plus the constraints of sensor resources, and then optimize the target function to obtain effective allocation of sensors to targets $[11-20]$. There are some different frameworks for the sensor management problem, such as the information-based approach [21-23], the posterior Cramér-Rao lower bound (PCRLB) approach $[11,24]$ and the covariance control approach [25-28]. The information-based approach only considers the amount of information rather than the actual value of information. They are designed to maximize the amount of information provided by the sensor, regardless of whether the resulting information is useful. Moreover, they cannot flexibly manage sensor resources based on the desired tracking accuracy, especially when sensor resources are plentiful. Compared with other sensor management approaches, the covariance control approach can easily impose a targetspecific covariance goal and balance tracking performance with sensor resources.

In this paper, the LEO infrared warning system is taken as the background. Based on the covariance control method, the general framework and specific algorithm for ballistic target tracking and multi-sensor management are established. The feasibility and superiority of the proposed method are verified in the simulation.

The following is a brief introduction. In Section 2, we describe the preliminaries of the paper. The covariance control approach is presented for a ballistic missile in Section 3. Section 4 presents some matrix metrics to measure the differentia between the desired covariance matrix and the actual covariance of each target. The efficiency and performance of the covariance control approach are proved by a case study in Section 5. Section 6 summarizes the full paper and looks forward to future work. 


\section{Preliminaries}

\subsection{Target motion model}

Suppose the state vector of missile target is $\boldsymbol{x}=$ $\left(x(t), y(t), z(t), v_{x}(t), v_{y}(t), v_{z}(t)\right)^{\mathrm{T}}$ in Earth centered fixed (ECF) coordinate system. In this case, the state-space model of a midcourse phase missile target has the following form [29-32]:

$$
\dot{\boldsymbol{x}}_{t}=f\left(\boldsymbol{x}_{t}\right)=
$$

$$
\left\{\begin{array}{l}
\dot{x}(t)=v_{x}(t) \\
\dot{y}(t)=v_{y}(t) \\
\dot{z}(t)=v_{z}(t) \\
\dot{v}_{x}(t)=-\frac{\mu}{r(t)^{3}}\left(1+\frac{c_{e}}{r(t)^{2}}\left(1-5 \sin ^{2}\left(\frac{z(t)}{r(t)}\right)\right)\right) . \\
x(t)+\omega_{e}^{2} \cdot x(t)+2 \omega_{e} \cdot v_{y}(t) \\
\dot{v}_{y}(t)=-\frac{\mu}{r(t)^{3}}\left(1+\frac{c_{e}}{r(t)^{2}}\left(1-5 \sin ^{2}\left(\frac{z(t)}{r(t)}\right)\right)\right) . \\
y(t)+\omega_{e}^{2} \cdot y(t)-2 \omega_{e} \cdot v_{x}(t) \\
\dot{v}_{z}(t)=-\frac{\mu}{r(t)^{3}}\left(1+\frac{c_{e}}{r(t)^{2}}\left(1-5 \sin ^{2}\left(\frac{z(t)}{r(t)}\right)\right)\right) . \\
z(t)-2 \frac{\mu \cdot c_{e}}{r(t)^{4}} \sin \left(\frac{z(t)}{r(t)}\right)
\end{array}\right.
$$

where $r(t)=\sqrt{x(t)^{2}+y(t)^{2}+z(t)^{2}}, \mu$ is Earth's gravitational constant and $\mu=3.9860064 \times 10^{5} \mathrm{~km}^{3} / \mathrm{s}^{2}, J_{2}$ is the second-order gravitational harmonic term of the Earth's gravitational field model and $J_{2}=-1.082626836 \times$ $10^{-3}, \omega_{e}$ is the Earth's angular velocity and $\omega_{e}=$ $7.292115 \times 10^{-5} \mathrm{rad} / \mathrm{s}, R_{e}$ represents the Earth's equatorial radius and $R_{e}=6378.14 \mathrm{~km}, c_{e}=3 J_{2} R_{e}^{2} / 2$.

\subsection{Infrared sensor measurement model}

In general, the space-based infrared sensor obtains the lineof-sight (LOS) measurement information of the target relative to the satellite by effectively suppressing the background clutter and using the contrast between the target and the background. In [32], we have developed the infrared sensor measurement model, as shown in Fig. 1.

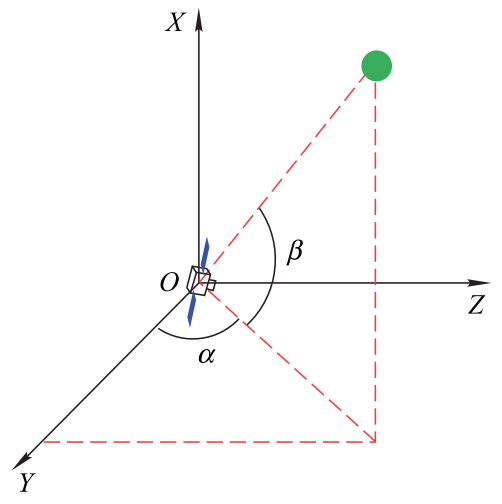

Fig. 1 Sensor measurement model
Suppose the target position vector in measurement coordinate system is $\boldsymbol{P}_{\mathrm{S}}=\left[x_{T}, y_{T}, z_{T}\right]^{\mathrm{T}}$, azimuth $\alpha$ and elevation $\beta$ can be calculated using

$$
\left(\begin{array}{c}
\alpha \\
\beta
\end{array}\right)=h\left(\boldsymbol{P}_{\mathrm{S}}\right)=\left(\begin{array}{c}
\arccos \left(\frac{y_{T}}{\sqrt{y_{T}^{2}+z_{T}^{2}}}\right) \\
-\arccos \left(\frac{x_{T}}{\sqrt{y_{T}^{2}+z_{T}^{2}}}\right)
\end{array}\right) .
$$

If $\boldsymbol{R}_{\mathrm{T}}$ and $\boldsymbol{R}_{\mathrm{S}}$ represent the target position vector and satellite position vector in ECF coordinate system, respectively, the relationship between measurement and target position can be expressed as

$$
\boldsymbol{y}=\left(\begin{array}{c}
\alpha \\
\beta
\end{array}\right)=h\left(\boldsymbol{M}_{\mathrm{E} \rightarrow \mathrm{S}}\left(\boldsymbol{R}_{\mathrm{T}}-\boldsymbol{R}_{\mathrm{S}}\right)\right)
$$

where $M_{\mathrm{E} \rightarrow \mathrm{S}}$ represents the transformation matrix from ECF to the measurement coordinate system.

\subsection{Target state estimation using EKF}

The extended Kalman filter (EKF) is very suitable for midcourse target tracking. In general, the discretized state and the measurement can be obtained as

$$
\left\{\begin{array}{l}
\boldsymbol{x}_{k}=f\left(\boldsymbol{x}_{k-1}\right)+\boldsymbol{w}_{k-1} \\
\boldsymbol{y}_{k}=h\left(\boldsymbol{x}_{k}\right)+\boldsymbol{v}_{k}
\end{array}\right.
$$

where $\boldsymbol{w}_{k-1}$ is the process noise and $\boldsymbol{v}_{k}$ is the sensor measurement error, and

$$
\left\{\begin{array}{l}
E\left(\boldsymbol{w}_{k}\right)=0 \\
E\left(\boldsymbol{w}_{k} \boldsymbol{w}_{j}^{\mathrm{T}}\right)=\boldsymbol{Q}_{k} \\
E\left(\boldsymbol{v}_{k}\right)=0 \\
E\left(\boldsymbol{v}_{k} \boldsymbol{v}_{j}^{\mathrm{T}}\right)=\boldsymbol{R}_{k} \\
E\left(\boldsymbol{w}_{k} \boldsymbol{v}_{j}^{\mathrm{T}}\right)=0
\end{array} .\right.
$$

First, after applying the first-order linearization, the state equation can be represented as the following form:

$$
\begin{gathered}
\boldsymbol{x}_{k}=\boldsymbol{x}_{k-1}+T \cdot \frac{\partial \boldsymbol{f}}{\partial \boldsymbol{x}_{k-1}} \cdot \boldsymbol{x}_{k-1} \\
\frac{\partial \boldsymbol{f}}{\partial \boldsymbol{x}_{k-1}}=\left.\frac{\partial f(\boldsymbol{x})}{\partial \boldsymbol{x}}\right|_{\boldsymbol{x}=\boldsymbol{x}_{k-1}}
\end{gathered}
$$

where $T$ is the sampling step.

According to (1),

$$
\frac{\partial f(\boldsymbol{x})}{\partial \boldsymbol{x}}=\left[\begin{array}{cc}
\mathbf{0}_{3 \times 3} & \boldsymbol{I}_{3 \times 3} \\
\boldsymbol{A} & \mathbf{0}_{3 \times 3}
\end{array}\right]
$$

where

$$
\boldsymbol{A}=\left[\begin{array}{lll}
\frac{\partial \ddot{x}}{\partial x} & \frac{\partial \ddot{y}}{\partial x} & \frac{\partial \ddot{z}}{\partial x} \\
\frac{\partial \ddot{x}}{\partial y} & \frac{\partial \ddot{y}}{\partial y} & \frac{\partial \ddot{z}}{\partial y} \\
\frac{\partial \ddot{x}}{\partial z} & \frac{\partial \ddot{y}}{\partial z} & \frac{\partial \ddot{z}}{\partial z}
\end{array}\right]
$$


The elements of $\boldsymbol{A}$ can be found in [32].

The state equation can be further simplified to the following form:

$$
\boldsymbol{x}_{k}=\boldsymbol{F}_{k} \cdot \boldsymbol{x}_{k-1}+\boldsymbol{w}_{k-1}
$$

where

$$
\begin{gathered}
\boldsymbol{F}_{k}=\boldsymbol{I}_{6 \times 6}+\frac{\partial f(\boldsymbol{x})}{\partial \boldsymbol{x}} \cdot T= \\
\boldsymbol{I}_{6 \times 6}+T \cdot\left[\begin{array}{cc}
\mathbf{0}_{3 \times 3} & \boldsymbol{I}_{3 \times 3} \\
\boldsymbol{A} & \mathbf{0}_{3 \times 3}
\end{array}\right]=\left[\begin{array}{cc}
\boldsymbol{I}_{3 \times 3} & T \cdot \boldsymbol{I}_{3 \times 3} \\
T \cdot \boldsymbol{A} & \boldsymbol{I}_{3 \times 3}
\end{array}\right] .
\end{gathered}
$$

Similarly, the observation equation becomes

$$
\boldsymbol{y}_{k}=\boldsymbol{H}_{k} \cdot \boldsymbol{x}_{k}+\boldsymbol{v}_{k}
$$

where $\boldsymbol{H}_{k}$ is the Jocabian matrix.

Therefore, EKF has two main steps.

Prediction step:

$$
\begin{gathered}
\widehat{\boldsymbol{x}}_{k, k-1}=f\left(\widehat{\boldsymbol{x}}_{k-1, k-1}\right) \\
\boldsymbol{P}_{k, k-1}=\boldsymbol{F}_{k-1} \boldsymbol{P}_{k-1, k-1} \boldsymbol{F}_{k-1}^{\mathrm{T}}+\boldsymbol{Q}_{k} .
\end{gathered}
$$

Innovation step:

$$
\begin{gathered}
\boldsymbol{K}_{k}=\boldsymbol{P}_{k, k-1} \boldsymbol{H}_{k}^{\mathrm{T}}\left[\boldsymbol{H}_{k} \boldsymbol{P}_{k, k-1} \boldsymbol{H}_{k}^{\mathrm{T}}+\boldsymbol{R}_{k}\right]^{-1} \\
\widehat{\boldsymbol{x}}_{k, k}=\widehat{\boldsymbol{x}}_{k, k-1}+\boldsymbol{K}_{k}\left[\boldsymbol{y}_{k}-h\left(\widehat{\boldsymbol{x}}_{k, k-1}\right)\right] \\
\boldsymbol{P}_{k, k}=\left[\boldsymbol{I}-\boldsymbol{K}_{k} \boldsymbol{H}_{k}\right] \boldsymbol{P}_{k, k-1} .
\end{gathered}
$$

\section{Sensor management algorithm with covariance control}

Covariance control starts from a desired covariance matrix, which is the main difference from other sensor management approaches. The sensor management algorithm with covariance control can be represented as the following form:

$$
D_{\mathrm{opt}}\left(t_{k}\right)=\arg \min _{D} f\left(\boldsymbol{P}_{i}\left(t_{k}\right), \boldsymbol{P}_{d}\left(t_{k}\right)\right)
$$

where $\boldsymbol{P}_{i}\left(t_{k}\right)$ is the actual covariance for the sensor combination $D_{i}$ at $t_{k} . \boldsymbol{P}_{d}\left(t_{k}\right)$ is the desired covariance at $t_{k}$. $f(\cdot, \cdot)$ is the matrix metric function. $D_{\text {opt }}\left(t_{k}\right)$ is the optimum sensor selection combination at $t_{k}$.

It also must be pointed out that sensors are only available when satellites are in the observation time window. In other words, the satellites we dispatch at any one time are those that are visible to the ballistic missile. The visibility analysis of LEO satellites and ballistic missiles has been discussed [33].

Assuming that there are $N_{s}(k)$ possible combinations that can be selected by the sensor scheduler at $t_{k}$. The main steps of the sensor management algorithm are:
Step 1 Initialize $i=1$;

Step 2 Calculate the prediction covariance $\boldsymbol{P}\left(t_{k}^{-}\right)$;

Step 3 Calculate the covariance $\boldsymbol{P}_{i}\left(t_{k}\right)$ with sensor combination $D_{i}$;

Step 4 Calculate $f\left(\boldsymbol{P}_{i}\left(t_{k}\right), \boldsymbol{P}_{d}\left(t_{k}\right)\right)$ based on some matrix metric $f(\cdot, \cdot)$;

Step $5 i=i+1$, if $i \leqslant N_{s}(k)$, go to Step 3;

Step 6 Select the sensor combination which minimizes $f\left(\boldsymbol{P}_{i}\left(t_{k}\right), \boldsymbol{P}_{d}\left(t_{k}\right)\right)$.

In order to achieve the purpose of controlling the tracking accuracy and dynamically adjust the sensor usage to meet the requirements of tracking accuracy, the covariance control approach draws on the theory of adaptive signal processing and generates feedback information by setting the desired covariance. The block diagram of the sensor management algorithm is shown in Fig. 2. The covariance controller is responsible for determining a sensor combination for each target to meet the desired covariance level, while the sensor scheduler prioritizes the sensing requests from the covariance controller and issues commands regarding which actions are to be executed during every scanning interval. The available sensors controlled by the sensor scheduler collect information from the external environment.

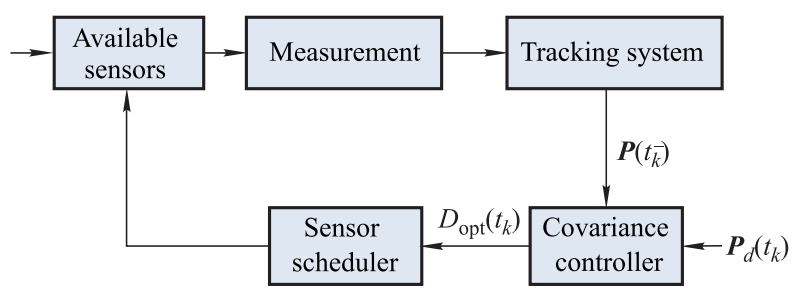

Fig. 2 Block diagram of the sensor management algorithm

\section{Matrix metrics}

Because the covariance control approach selects sensor combinations based on the difference between the desired covariance matrix and the actual covariance of each target, we need to use some matrix metrics to measure the differentia between two covariance matrices [32,34]. Equations (19) - (21) give the three most commonly used matrix measures, and we choose the trace metric in this paper.

M1: Absolute trace metric

$$
f(\boldsymbol{A}, \boldsymbol{B})=\operatorname{tr}(|\boldsymbol{M}|)=\sum_{i=1}^{n}\left|m_{i i}\right|
$$

where $\boldsymbol{M}=\boldsymbol{A}-\boldsymbol{B}$.

M2: 2-norm metric

$$
f(\boldsymbol{A}, \boldsymbol{B})=\|\boldsymbol{M}\|_{2}=\sigma_{1}(\boldsymbol{M})
$$

where $\sigma_{1}$ denotes the maximum eigenvalue of the matrix $M$. 
M3: determinant metric

$$
f(\boldsymbol{A}, \boldsymbol{B})=\operatorname{det}(\boldsymbol{M})
$$

Under the Gaussian assumption, the covariance matrix physically represents an ellipsoid bounding the likely target state values whose probability exceeds a threshold. The lengths of the axes for the ellipsoid are proportional to the eigenvalues of the covariance matrix. The trace of the covariance matrix is the overall expected mean squared error (MSE) for the state estimator (or the sum of the length of the axes, while the maximum eigenvalue represents the length of the major axis), and the determinant is the volume of the ellipsoid. In these cases, the goal is to reduce the measure error, which in turn reduces the size of the ellipsoid. We consider the two-dimensional (2-D) scenario to facilitate the problem description. The desired covariance ellipse and the actual covariance ellipse are shown in Fig. 3. In Fig. 3(a), the two ellipses are of the same size, but $\boldsymbol{P}_{i}\left(t_{k}\right)$ does not meet the requirement of $\boldsymbol{P}_{d}\left(t_{k}\right)$. In fact, as shown in Fig. 3(b), the ellipse formed by $\boldsymbol{P}_{d}\left(t_{k}\right)$ will completely enclose the ellipse formed by $\boldsymbol{P}_{i}\left(t_{k}\right)$, which ensures that the actual covariance $\boldsymbol{P}_{i}\left(t_{k}\right)$ is within the desired covariance $\boldsymbol{P}_{d}\left(t_{k}\right)$.

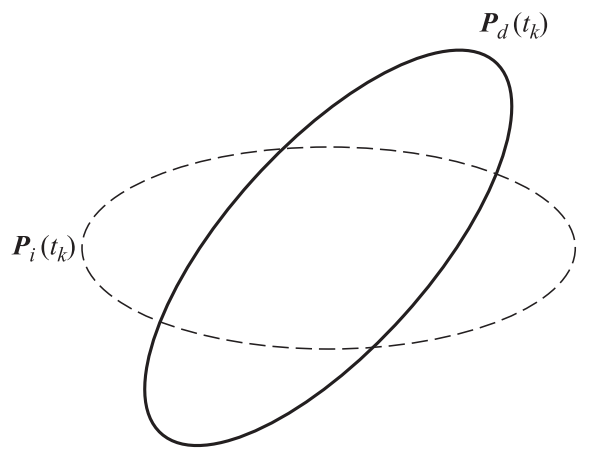

(a) Ellipses are of the same size but represent very different covariance matrices

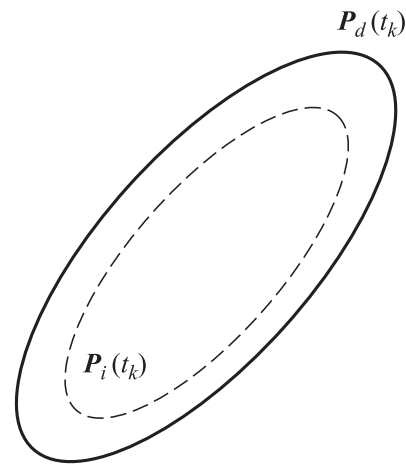

(b) Ellipse formed by the desired covariance encloses that formed by the actual covariance (modified from [25])

Fig. 3 Desired covariance ellipse and the actual covariance ellipse
Theoretically, if the covariance difference $\boldsymbol{P}_{d}\left(t_{k}\right)-$ $\boldsymbol{P}_{i}\left(t_{k}\right)$ is the positive semidefinite, then the ellipsoid formed by $\boldsymbol{P}_{i}\left(t_{k}\right)$ is enclosed by that of $\boldsymbol{P}_{d}\left(t_{k}\right)$. However, this limitation is actually too strict in practice, especially for the ballistic target tracking, in which scenario the satellites and targets are moving fast. In this paper, we believe that the desired covariance $\boldsymbol{P}_{d}\left(t_{k}\right)$ has been met even though the difference $\boldsymbol{P}_{d}\left(t_{k}\right)-\boldsymbol{P}_{i}\left(t_{k}\right)$ is not positive semidefinite (the minimum eigenvalue is negative), as long as the difference is small. In other words, selecting the optimal subset of sensors brings the actual covariance closest to the desired covariance.

One important issue that has not been discussed is the unit system problem. For the state of a 2-D target, the covariance may have the following units:

$$
\boldsymbol{P}_{i}\left(t_{k}\right)=\left[\begin{array}{cc}
\mathrm{m}^{2} & \mathrm{~m}^{2} / \mathrm{s} \\
\mathrm{m}^{2} / \mathrm{s} & \mathrm{m}^{2} / \mathrm{s}^{2}
\end{array}\right] .
$$

The different units can make the interpretation of some metrics difficult [34]. Using the trace metric will result in a sum of elements with units of $\mathrm{m}^{2}$ and $\mathrm{m}^{2} / \mathrm{s}^{2}$. This effect caused by different units can be eliminated by using a transformation matrix similar to that in [35].

$$
\begin{gathered}
\widetilde{\boldsymbol{P}}_{i}\left(t_{k}\right)=\boldsymbol{L} \boldsymbol{P}_{i}\left(t_{k}\right) \boldsymbol{L}= \\
{\left[\begin{array}{ll}
1 & 0 \\
0 & \mathrm{~s}
\end{array}\right]\left[\begin{array}{cc}
\mathrm{m}^{2} & \mathrm{~m}^{2} / \mathrm{s} \\
\mathrm{m}^{2} / \mathrm{s} & \mathrm{m}^{2} / \mathrm{s}^{2}
\end{array}\right]\left[\begin{array}{cc}
1 & 0 \\
0 & \mathrm{~s}
\end{array}\right]}
\end{gathered}
$$

The unit of each element in matrix $\widetilde{\boldsymbol{P}}_{i}\left(t_{k}\right)$ is $\mathrm{m}^{2}$.

In addition to the above mentioned approach, we can use another unitless transformation approach [25].

$$
\begin{gathered}
\widetilde{\boldsymbol{P}}_{i}\left(t_{k}\right)=\boldsymbol{L} \boldsymbol{P}_{i}\left(t_{k}\right) \boldsymbol{L}= \\
{\left[\begin{array}{cc}
1 / \sigma_{x} & 0 \\
0 & 1 / \sigma_{v x}
\end{array}\right]\left[\begin{array}{cc}
\mathrm{m}^{2} & \mathrm{~m}^{2} / \mathrm{s} \\
\mathrm{m}^{2} / \mathrm{s} & \mathrm{m}^{2} / \mathrm{s}^{2}
\end{array}\right]\left[\begin{array}{cc}
1 / \sigma_{x} & 0 \\
0 & 1 / \sigma_{v x}
\end{array}\right]}
\end{gathered}
$$

where $\sigma_{x}^{2}$ and $\sigma_{v x}^{2}$ are the diagonal elements of $\boldsymbol{P}_{d}\left(t_{k}\right)$.

In the target tracking process, we are most concerned about the position estimation accuracy. For the sake of simplicity, the example presented here only considers the position terms and we do not need to consider the unit problem.

\section{Application and verification of covariance control approach}

The Walker- $\delta$ constellation is used as the background. The constellation and the target parameters can be found in [32]. Suppose that 24 satellites work synchronously, and the sampling step is $1 \mathrm{~s}$. Each time at least two satellites are needed to observe the target because of the bearingsonly measurements. However, at any one time, up to four satellites can be used because of the physical limitations. 
The bearing error standard deviation of the target generated measurements is $\sigma_{\mathrm{LOS}}=90 \mu \mathrm{rad}$. In order to show the covariance control ability and performance of the proposed covariance control approach, the desired covariance matrix is starting as a diagonal matrix with eigenvalues [10 000, $10000,10000,100,100,100]$ and decreasing to a diagonal matrix with eigenvalues [2000, 2000,2000 , $10,10,10]$ at $150 \mathrm{~s}$. All algorithms are coded in Matlab and run on an Intel $/ 3.4 \mathrm{GHz}$ processor.

Figs. 4-6 show the desired covariance and the actual covariance over time by the covariance control approach. As can be seen from Figs. 4-6, it is clear that the actual covariance has been successfully controlled around the desired covariance. This fact indicates that the covariance control approach has a good performance for maintaining a specific desired covariance for a missile target.

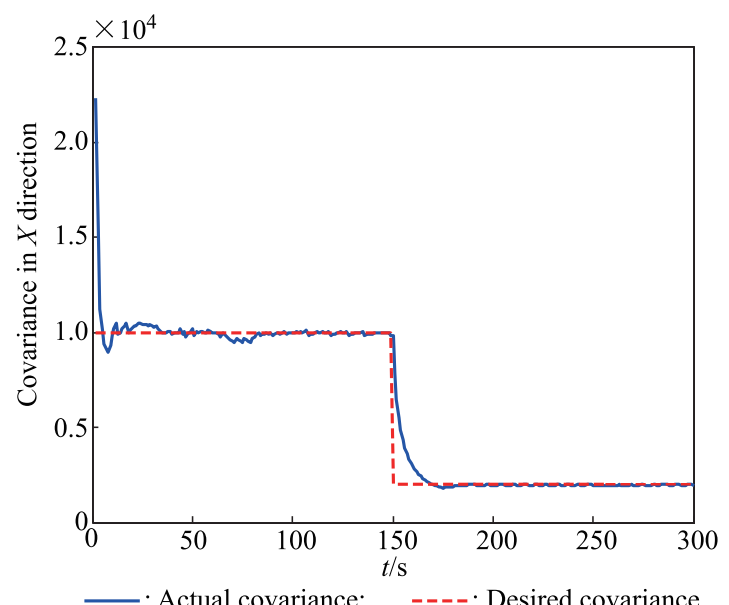

Fig. 4 Desired covariance and actual covariance in $X$ direction

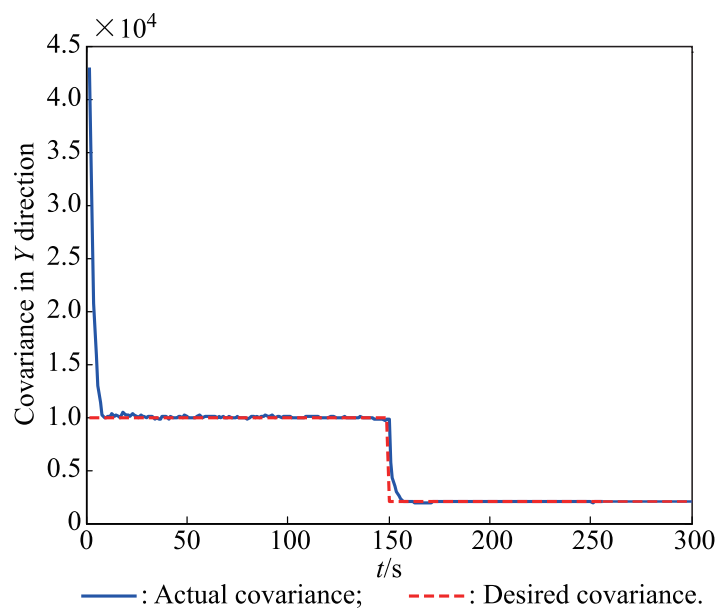

Fig. 5 Desired covariance and actual covariance in $Y$ direction

For the purpose of effectively illustrating the advantages of our proposed method, a performance comparison is implemented with other two "unmanaged" systems that ran- domly select two or four satellites each time. 50 Monte Carlo runs are performed.

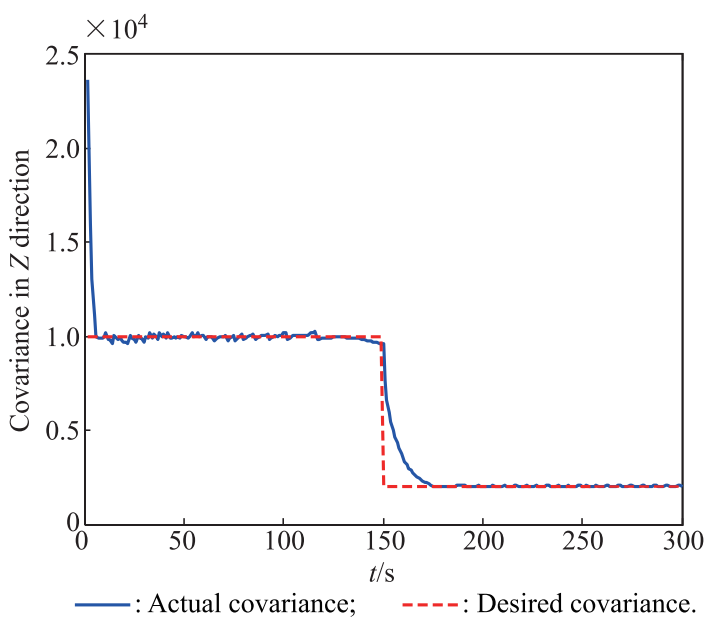

Fig. 6 Desired covariance and actual covariance in $Z$ direction

Fig. 7 shows the comparison of performance in terms of root mean square error (RMSE). Comparing the simulation results with the two satellites unmanaged system, it can be seen that the covariance control approach can obtain a better tracking performance. In addition, Fig. 7 also shows that the covariance control approach leads to a higher tracking accuracy than the four satellites unmanaged systems after $150 \mathrm{~s}$. The findings demonstrate that the covariance control approach is able to provide a better tracking performance by providing a well-designed desired covariance, even though the unmanaged system uses all its satellites resources.

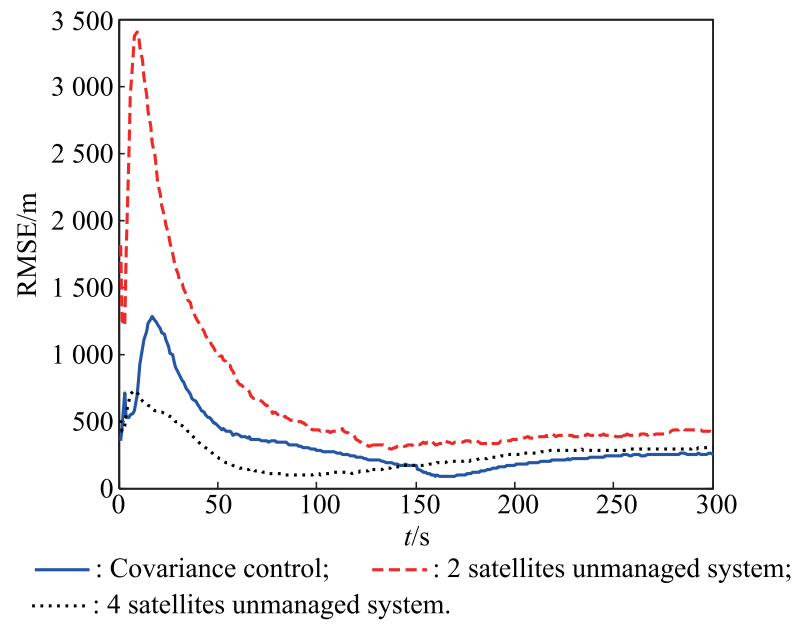

Fig. 7 Comparison of performance in terms of the RMSE

Fig. 8 and Fig. 9 show which satellites are chosen and the number of satellites used during the tracking task, respectively. With reference to Figs. 4-6, four satellites are needed at the initial stage to quickly converge the actual covariance to the desired covariance. It can be seen that 
in the first $150 \mathrm{~s}$, the covariance control approach uses fewer sensors than four satellites unmanaged system and uses more sensors than two satellites unmanaged system. The LEO constellation consumes more sensors resources than the two satellites unmanaged system at the beginning of the target tracking because of the large initial error and the need to quickly reduce the tracking error by using as many sensors as possible. When entering the stable tracking phase, the LEO constellation only needs to use two satellites. During $150 \mathrm{~s}$ to $300 \mathrm{~s}$, because of the more restrictive constraints, the covariance control approach almost uses all of its sensors that can be used synchronously to achieve the more demanding desired covariance. While the four satellites unmanaged system wastes satellites resources by selecting all satellites each time, the covariance control approach can effectively balance tracking performance goals with system requirements, allocating maximum resources only when necessary.

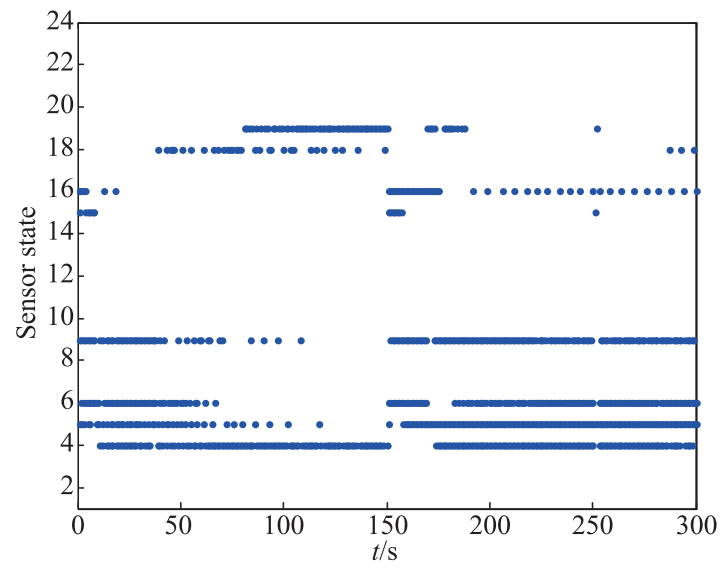

Fig. 8 Sensor state during the tracking task

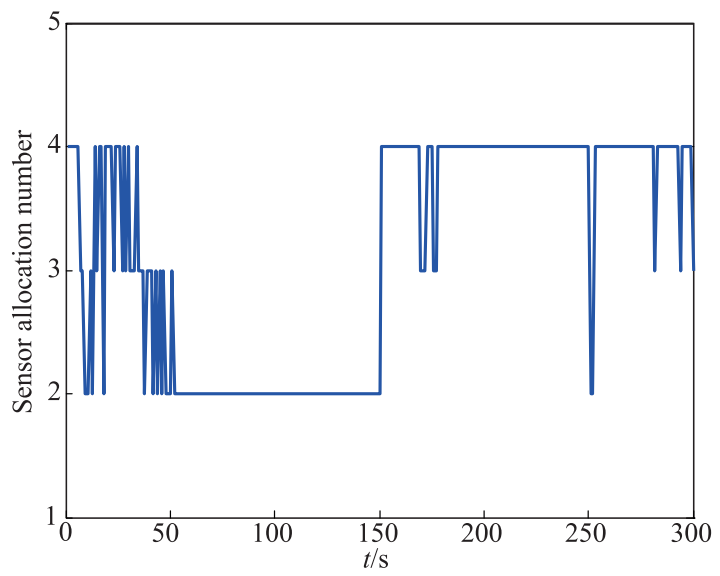

Fig. 9 Sensor allocation number during the tracking task

Fig. 8 and Fig. 9 also show the defects of the original covariance control approach. According to Fig. 8 and Fig. 9, it is clear that the covariance control approach may cause frequent switching in LEO constellation. In order to measure the size of the switching frequency, we define a parameter called the switching frequency that is calculated as the ratio of the quantity of switching in adjacent time divided by the total number of selection during the tracking task. The switching frequency is 0.52 . In engineering practice, such a big switching frequency can bring many negative consequences and needs to be improved [32].

There is no correlation between the choice of sensor combinations at each moment. As a result, the combination of sensors used in adjacent time is usually different. In order to reduce the switching frequency under the premise of ensuring tracking performance, we can improve the original covariance control approach by designing a predefined boundary $\delta$. The improved covariance control approach relaxes the demands of the matrix trace metric, allowing the trace of the covariance difference to vary between the range of $\pm \delta$.

Suppose $\delta=2$ 000. Fig. 10 and Fig. 11 show which satellites are chosen and the number of satellites used during the tracking task when $\delta=2000$, respectively.

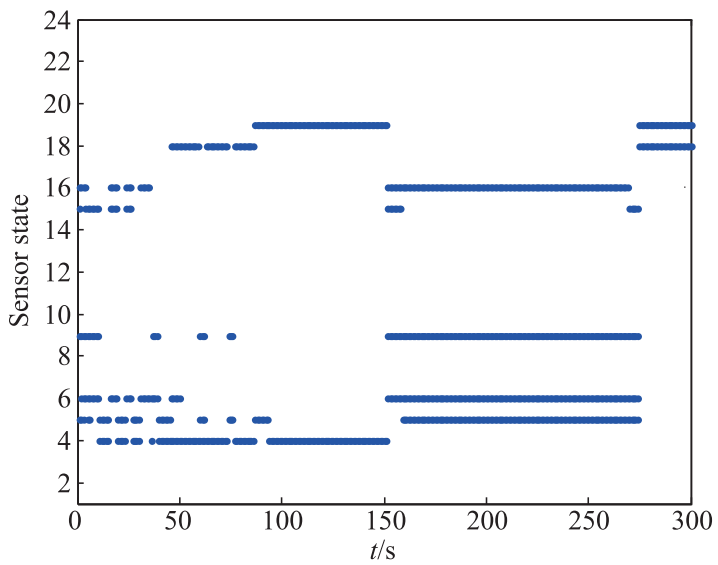

Fig. 10 Sensor state during the tracking task after improving the covariance control approach

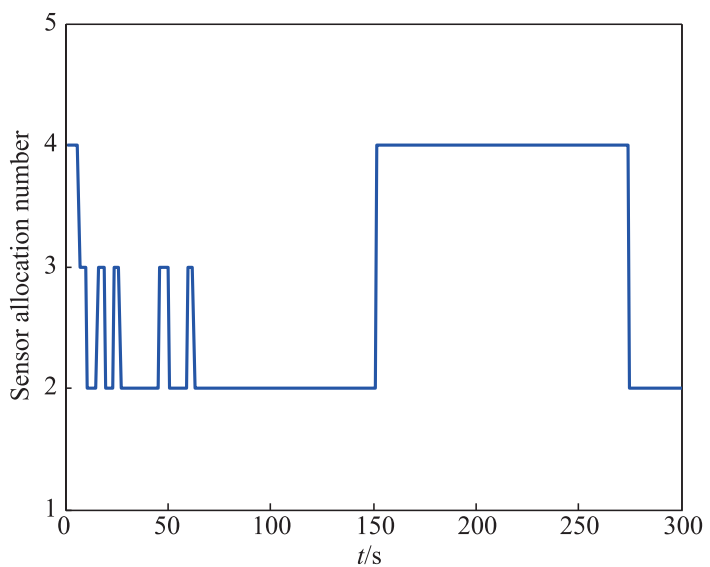

Fig. 11 Sensor allocation number during the tracking task after improving the covariance control approach 
The switching frequency is 0.08 . As can be seen from Fig. 10 and Fig. 11, the improved covariance control approach has successfully reduced switching frequency significantly.

Figs. $12-14$ show the desired covariance and the actual covariance over time by the improved covariance control approach. According to Figs. $12-14$, it is clear that the actual covariance has been successfully controlled around the desired covariance. Compared to the original covariance control approach, the actual covariance obtained by the improved covariance control approach fluctuates around the desired covariance. In detail, compared to Fig. 4, Fig. 12 shows that the $X$ direction covariance control accuracy of improved covariance control approach is reduced, and the maximum deviation from the desired covariance (without considering the initial tracking phase and the desired covariance changing phase) is about 1687 , which does not exceed the predefined boundary.

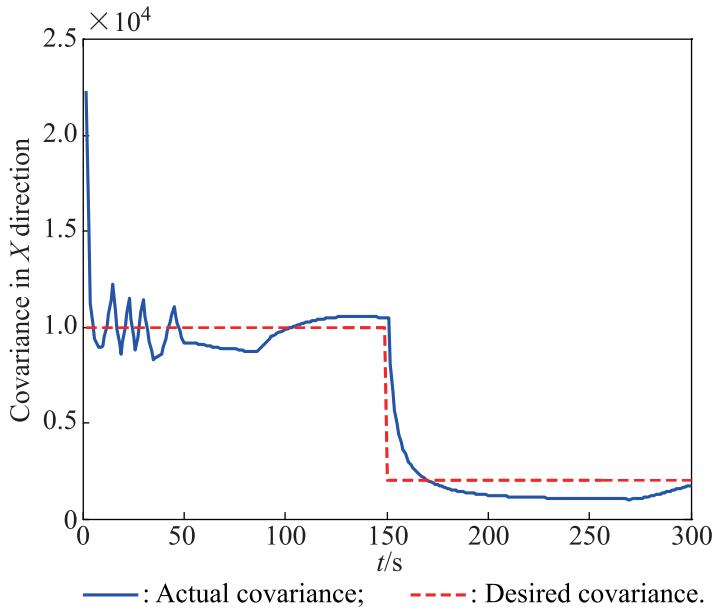

Fig. 12 Desired covariance and actual covariance in $X$ direction after improving the covariance control approach

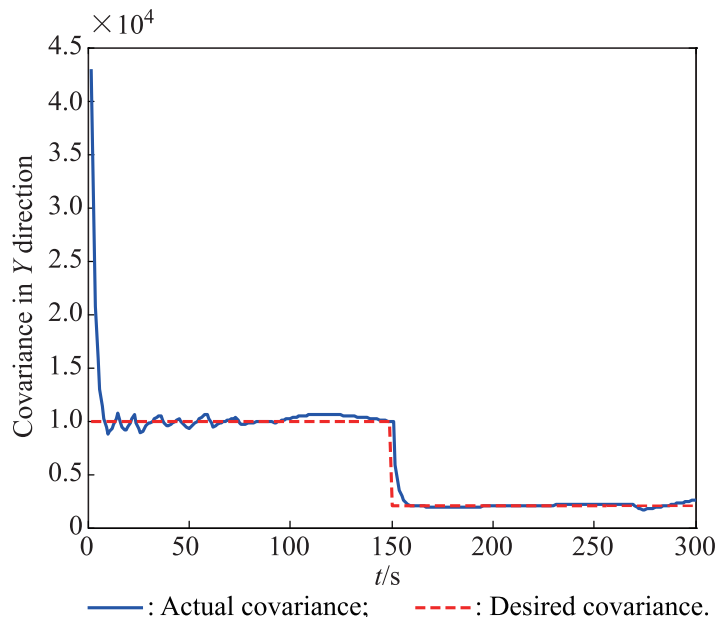

Fig. 13 Desired covariance and actual covariance in $Y$ direction after improving the covariance control approach

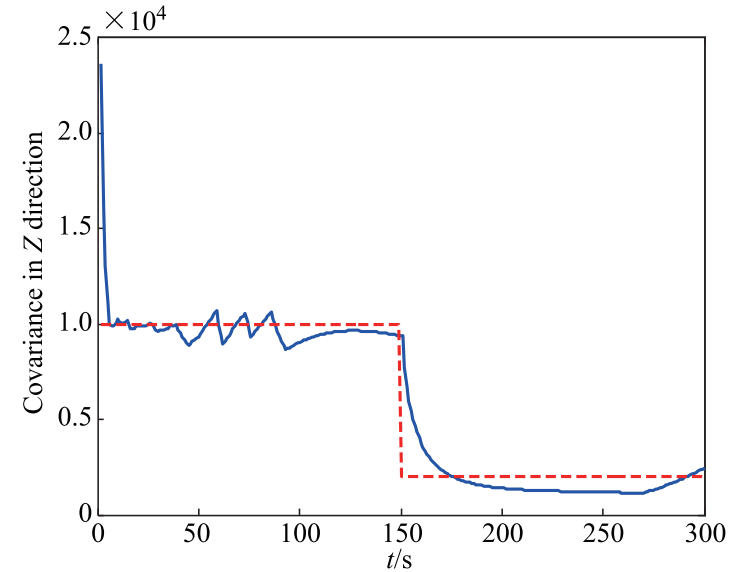

Fig. 14 Desired covariance and actual covariance in $Z$ direction after improving the covariance control approach

Compared to Fig. 5, Fig. 13 shows that the $Y$ direction covariance control accuracy of the improved covariance control approach is slightly reduced, and the maximum deviation from the desired covariance is about 645, which does not exceed the predefined boundary. Compared to Fig. 6, Fig. 14 shows that the $Z$ direction covariance control accuracy of the improved covariance control approach is reduced, and the maximum deviation from the desired covariance is about 1362 , which does not exceed the predefined boundary. However, the decrease of covariance control accuracy does not mean that the tracking performance deteriorates. We will confirm this judgment in the next simulation.

Fig. 15 shows the comparison of performance between the covariance control method and the improved covariance control method. We can find that the tracking performance has been improved significantly. The average RMSE of the original covariance control approach and the improved covariance control approach are $327.985 \mathrm{~m}$ and $259.383 \mathrm{~m}$, respectively. That is to say, a $20.9 \%$ performance improvement is obtained if we use the improved covariance control approach.

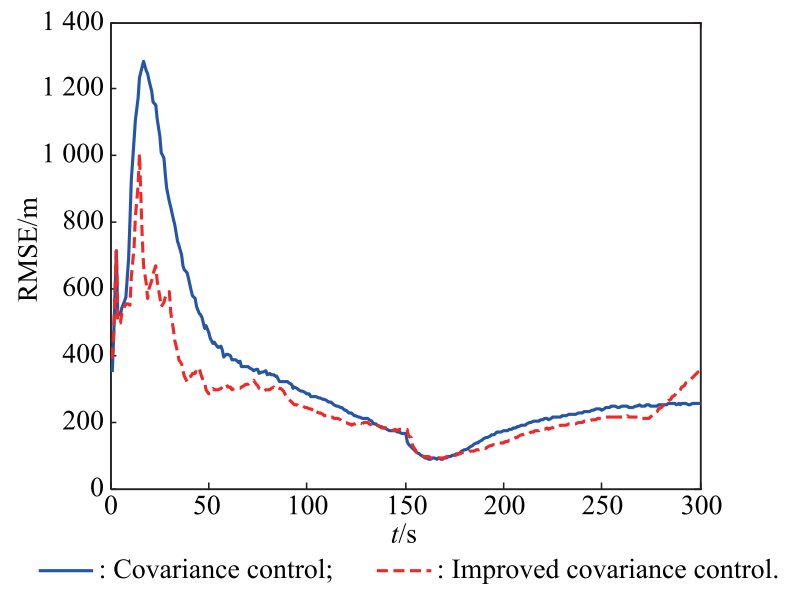

Fig. 15 Comparison of performance in terms of the RMSE 
Therefore, the proposed improved covariance control method can significantly reduce the switching frequency and improve the tracking performance. The findings demonstrate that a big switching frequency may bring some negative consequences and cause the deterioration of tracking accuracy, and the improved covariance control approach is able to provide a better performance by considering tradeoffs between the covariance control ability and the sensors switching frequency of the LEO constellation.

Fig. 16 shows the switching frequency versus predefined boundary. In general, the switching frequency and the covariance control ability of the tracking system will decrease along with the predefined boundary growth. When the predefined boundary is large enough, the switching frequency is reduced to zero, which means that the tracking system will use the same satellites all the time in the process of tracking a ballistic missile and lose the covariance control ability completely. Thus, in engineering practice, the predefined boundary should be designed according to the system requirements and be considered an optimization indicator if possible [32].

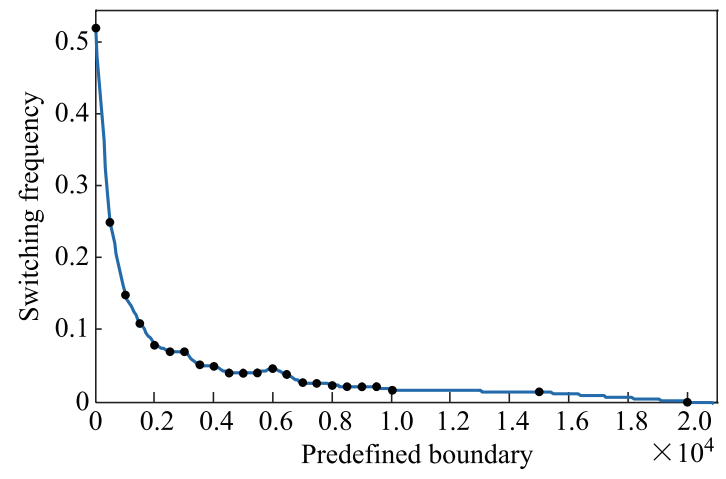

Fig. 16 Switching frequency versus predefined boundary

The average computational times (one step) of a sensor subset selection using the original covariance control approach and the improved covariance control approach are $0.56 \mathrm{~s}$, and $0.07 \mathrm{~s}$, respectively. Both methods can meet the real-time requirements. In addition, the improved covariance control approach has successfully improved the real-time performance significantly.

\section{Conclusions and future work}

This study aims at solving the sensor management of the LEO infrared constellation. The proposed covariance control approach is able to maintain a target's state estimate covariance near a specific desired level without overtaxing the sensor resources of the LEO infrared constellation. Simulation results demonstrate that the proposed covariance control approach outperform the unmanaged systems.

In this paper, we only consider the single target tracking. For this single target tracking problem, exhaustive enumeration of all sensor combinations is feasible, which allows real-time capability. However, complete enumeration is not feasible for multiple targets tracking problem because of the exponential growth of possible sensor combinations. Therefore, sensor management problem will become more complicated with multiple targets tracking problem. Furthermore, we do not consider the complicated data association problem in ballistic missile tracking. For the above two problems, we will make some efforts in the future.

\section{References}

[1] DAVIS M T, ROBBINS J M, LUNDAY B J. Approximate dynamic programming for missile defense interceptor fire control. European Journal of Operational Research, 2017, 259(3): $873-886$.

[2] BHATTACHARYYA A, SARASWAT V K, MANIMARAN $\mathrm{P}$, et al. Evidence theoretic classification of ballistic missiles. Applied Soft Computing, 2015, 37: 479-489.

[3] SINGH U K, PADMANABHAN V, AGARWAL A. Dynamic classification of ballistic missiles using neural networks and hidden Markov models. Applied Soft Computing, 2014, 19: $280-289$.

[4] GARCIA E, CASBEER D W, PACHTER M. Active target defense using first order missile models. Automatica, 2017, 78: $139-143$.

[5] PARNELL G S, METZGER R E, MERRICK J, et al. Multiobjective decision analysis of theater missile defense architectures. Systems Engineering, 2015, 4(1): 24-34.

[6] XIE X, LI W M, HUANG R Q. Optimization of space-based infrared satellites deployment for near-space hypersonic target warning. Laser and Infrared, 2015, 45(8): 924-930. (in Chinese)

[7] WU Z R, BAI G Z. Analysis of US ballistic missile warning and recognition technology development. Journal of Spacecraft TT\&C Technology, 2016, 35(6): 415-421. (in Chinese)

[8] ZHAO X S, WANG H X, CAI Y C. Resource scheduling method in antimissile early warning campaign. Systems Engineering and Electronics, 2015, 37(6): 1300-1305. (in Chinese)

[9] REN J L, XING Q H. Research on optimal deployment of detecting multi-target early-warning GBRs in missile defense system. Computer Engineering \& Applications, 2014, 50(11): 233 - 236. (in Chinese)

[10] PARK S, ROTHROCK L. Systematic analysis of framing bias in missile defense: implications toward visualization design. European Journal of Operational Research, 2007, 182(3): $1383-1398$.

[11] THARMARASA R, KIRUBARAJAN T, HERNANDEZ M L. Large-scale optimal sensor array management for multitarget tracking. IEEE Trans. on Systems Man \& Cybernetics, Part C (Applications and Reviews), 2007, 37(5): 803-814.

[12] ZHANG Y, CAO N, WIMALAJEEWA T, et al. Compressive sensing based probabilistic sensor management for target tracking in wireless sensor networks. IEEE Trans. on Signal Processing, 2015, 63(22): 6049-6060.

[13] GOSTAR A K, HOSEINNEZHAD R, BAB-HADIASHAR A. Robust multi-Bernoulli sensor selection for multi-target tracking in sensor networks. IEEE Signal Processing Letters, 2013, 20(12): $1167-1170$.

[14] SOUZA F L, NAKAMURA E F, PAZZI R W. Target tracking for sensor networks: a survey. ACM Computing Surveys, 
2016, 49(2): $1-31$.

[15] BHUIYAN M Z A, WANG G, VASILAKOS A V. Local area prediction-based mobile target tracking in wireless sensor networks. IEEE Trans. on Computers, 2015, 64(7): 1968-1982.

[16] WANG T, PENG Z, LIANG J, et al. Following target for mobile tracking in wireless sensor networks. ACM Trans. on Sensor Networks, 2016, 12(4): 1-24.

[17] SHI K, CHEN K, LIN Y. Probabilistic coverage based sensor scheduling for target tracking sensor networks. Information Sciences, 2015, 292(C): 95-110.

[18] SHI J, WANG F, ZHOU J. A novel sensor selection and power allocation algorithm for multiple-target tracking in an LPI radar network. Sensors, 2016, 16(12): 2193-2206.

[19] HILAL A R, BASIR O A. A scalable sensor management architecture using BDI model for pervasive surveillance. IEEE Systems Journal, 2017, 9(2): 529-541.

[20] KASHI S S. A load balanced location service for location information management of multi-sink wireless sensor networks. Computing, 2017, 100(2): 1-25.

[21] KOLBA M P, SCOTT W R, COLLINS L M. A framework for information-based sensor management for the detection of static targets. IEEE Trans. on Systems, Man, and Cybernetics Part A: Systems and Humans, 2011, 41(1): 105-120.

[22] KOLBA M P, COLLINS L M. Information-based sensor management in the presence of uncertainty. IEEE Trans. on Signal Processing, 2007, 55(6): $2731-2735$.

[23] LUO W T, XU Y S, XIANG J J, et al. Sensor management based on random set observations and information theory. Modern Radar, 2013, 35(12): 38 -42. (in Chinese)

[24] PUNITHAKUMAR K, KIRUBARAJAN T, HERNANDEZ M. Multisensor deployment using PCRLBs, incorporating sensor deployment and motion uncertainties. IEEE Trans. on Aerospace and Electronic Systems, 2006, 42(4): 1474-1485.

[25] KALANDROS M, PAO L Y. Covariance control for multisensor systems. IEEE Trans. on Aerospace and Electronic Systems, 2002, 38(4): $1138-1157$.

[26] KALANDROS M, PAO L Y. Covariance control for sensor management in cluttered tracking environments. Journal of Guidance, Control \& Dynamics, 2004, 38(4): 493 - 496.

[27] KALANDROS M, PAO L Y. Multisensor covariance control strategies for reducing bias effects in interacting target scenarios. IEEE Trans. on Aerospace and Electronic Systems, 2005, 41(1): $153-173$.

[28] ZHOU W H, LI L, HU W D, et al. Performance evaluation of sensor allocation algorithm based on covariance control. Journal of Systems Engineering and Electronics, 2005, 16(3): $538-544$

[29] MENON P K A, BRIGGS M. Near-optimal midcourse guidance for air-to-air missiles. Journal of Guidance, Control and Dynamics, 2015, 13(4): 596-602.
[30] IMADO F, KURODA T, MIWA S. Optimal midcourse guidance for medium-range air-to-air missiles. Journal of Guidance, Control and Dynamics, 2015, 13(4): 603-608.

[31] QIN W, WANG X, CUI N. Maximum correntropy sparse Gauss-Hermite quadrature filter and its application in tracking ballistic missile. IET Radar, Sonar and Navigation, 2017, 11(9): $1388-1396$.

[32] QIN Z, LIANG Y G. Sensor management of LEO constellation using modified binary particle swarm optimization. Optik, 2018, 172: 879-891.

[33] TANG S X. Research on satellite sensor resources management and detection tasks scheduling problem for LEO constellation of space-based early warning system. Changsha: National University of Defense Technology, 2011. (in Chinese)

[34] YANG C, KAPLAN L, BLASCH E. Performance measures of covariance and information matrices in resource management for target state estimation. IEEE Trans. on Aerospace and Electronic Systems, 2012, 48(3): 2594-2613.

[35] HINTZ K J, MCVEY E S. Multi-process constrained estimation. IEEE Trans. on Systems, Man, and Cybernetics, 1991, 21(1): $237-244$.

\section{Biographies}

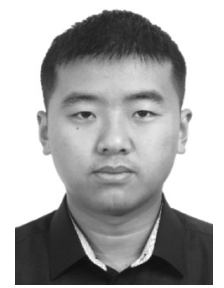

QIN Zheng was born in 1992. He received his B.S. degree in aerospace science and technology from Harbin Institute of Technology, China, in 2014, and his M.S. degree from National University of Defense Technology, China, in the area of aerospace science and technology in 2016. He is currently a Ph.D. candidate in the College of Aerospace Science and Engineering, National University of Defense Technology. His research interests include multitarget tracking, sensor management and satellite constellation scheduling.

E-mail: qinzheng911@126.com

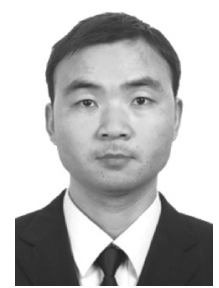

LIANG Yan'gang was born in 1979. He received his Ph.D. degree in aerospace science and technology from National University of Defense Technology, China, in 2009. He is currently an associate professor in the College of Aerospace Science and Engineering, National University of Defense Technology. His research interests include aerospace system analysis and simulation, and effectiveness assessment.

E-mail: liangyg@nudt.edu.cn 